\title{
CD10 expression in gastric carcinoma is correlated with tumor grade and survival
}

\author{
Amir Hosein Jafarian*, Melika Kooshki Forooshani**, Leila Takallou**, \\ and Nema Mohamadian Roshan**
}

\begin{abstract}
\section{BACKGROUND}

Gastric carcinoma (GC) is the most common non-skin malignancy in Iranian men and the second leading cause of cancer-related mortality. Invasion and metastasis are considered as the major causes of cancer-related morbidity and mortality. Proteinases such as matrix metalloproteinases play an important role in tumor progression and mediating extracellular matrix remodeling. CD10 is a $90-110 \mathrm{kd}$ cell surface zinc-dependent metalloproteinase and there is evidence that this membrane protein may facilitate invasion and/or metastasis of tumoral cells. The objective of this study was to determine the frequency of CD10 expression in the stromal cells of GC and determine its relationship with survival and clinicopathological factors.
\end{abstract}

\section{METHODS}

A cross-sectional study was performed involving 50 patients with histopathologic diagnosis of GC. CD10 expression was determined by immunohistochemistry (IHC). Survival of the patients as well as the grade and stage of the tumors and demographic variables were documented. The Kaplan-Meier test was used for data analysis.

\section{RESULTS}

Stromal CD10 was detected in $46 \%$ of the GC stromal cells. No immunoreactivity was identified in the stromal cells of normal adjacent tissue. Stromal CD10 expression in gastric carcinoma did not correlate with the age and gender of the cases as well as the size and location of the tumor, and lymph node involvement but correlated with tumor stage $(\mathrm{p}=0.01)$, tumor $\operatorname{grade}(\mathrm{p}=0.01)$ and patients' survival $(\mathrm{p}=0.02)$.

\section{CONCLUSION}

Stromal CD10 expression is correlated with tumor differentiation, clinical stage and survival in GC. CD10 expression could be considered as a negative prognostic factor for gastric carcinoma.

Keywords: CD10, immunohistochemistry staining (IHC), gastric carcinoma, stromal cell
*Cancer Molecular Pathology

Research Center,

Department of Pathology,

Faculty of Medicine, Mashhad University of Medical

Sciences, Mashhad, Iran

**Department of Pathology,

Faculty of Medicine,

Mashhad University of Medical

Sciences, Mashhad, Iran

\section{Correspondence}

Nema Mohamadian Roshan, MD

Pathology Department,

School of Medicine,

Mashhad University of Medical

Sciences, Mashhad, Iran

Email:roshann@mums.ac.ir

ORCID ID : 0000-0002-7862-7140

Date of first submission, December 14, 2018

Date of final revised submission, March 27, 2019

Date of acceptance, March 28, 2019

This open access article is distributed under a Creative Commons AttributionNon Commercial-Share Alike 4.0

International License

Cite this article as: Jafarian $\mathrm{AH}$, Forooshani MK Takallou L, et al. CD10 expression in gastric carcinoma is correlated with tumor grade and survival. Univ Med 2019;38:41-7. doi: 10.18051/UnivMed. 2019.v38.41-47 


\section{INTRODUCTION}

Gastric carcinoma (GC) is considered a deadly tumor and is the second most common cause of cancer-associated mortality. ${ }^{(1)}$ Approximately 990,000 patients are diagnosed with GC worldwide annually, of whom about 738,000 die from this malignancy. ${ }^{(2)}$ Gastric carcinoma also causes one of the highest cancer burdens, as measured by disability-adjusted life years lost. ${ }^{(3,4)}$

CD10, a common zinc-dependent metalloendoprotease that cleaves and inactivates a variety of signaling peptides, is now widely known for its involvement in various cancers. Its role in tumor progression, however, seems to differ in a tissue- or cancer-specific manner. ${ }^{(5)}$ In addition, previous studies on lymphoma, lung adenocarcinoma and invasive ductal carcinoma of the breast have shown that the expression of CD10 in primary tumors can serve as an independent predictor of poor prognosis.

For example in one study, it was demonstrated that CD10 expression in melanoma increased with tumor progression and predicted poor patient survival. ${ }^{(6)}$ Moreover, CD10 could have been widely used in cancer diagnosis while it recently has been proposed that CD10 is associated with therapeutic resistance. ${ }^{(7)}$

Recent research, focusing on the importance of stromal cells in pancreatic carcinomas, showed that pancreatic stellate cells which expressed CD10 were related to more aggressive nature. It has been suggested that CD10 could be a target for the treatment of pancreatic carcinomas using agents that inhibit metalloproteinase. For instance, fish oil which has some inhibitory effects on metalloproteinase, has been found useful in this regard. ${ }^{(8)}$

In a recent study, Murakami et al. ${ }^{(9)}$ tried to elucidate the clinicopathologic and immunohistochemical features of gastric adenocarcinoma with enteroblastic differentiation (GAED), by analyzing 29 cases. Immunohistochemistry for alpha-feto protein
(AFP), glypican 3, SALL4, and p53 and immunostaining with antibodies against MUC5AC, MUC6, MUC2, CD10, and caudaltype homeobox 2 (CDX2) were performed. CD10 was diffusely or focally expressed in all GAED cases while invasive areas with hepatoid or enteroblastic differentiation were negative for CD10, but they did not study the correlation between the mentioned items and the prognostic clinical factors of patients namely age, stage and grade of disease. This is what has been approached by our study.

Another study on CD10 revealed that CD10 down expression was frequently observed along with an additional distinct feature of gastrointestinal follicular lymphoma (GI-FL). CD10-downexpressed gastrointestinal follicular lymphoma (CD10 down GI FL) frequently involved the stomach or large intestine. However, there was no data on gastric carcinoma. ${ }^{(10)}$

In our study, not only the pathological features of the tumor (stage, grade, lymph node involvement) but also the survival was investigated. Moreover, size and location of the tumor were also considered in comparison with other similar studies. ${ }^{(11)}$

In this regard, we investigated whether CD10 expression predicted GC prognosis and whether it correlated with other clinicopathological factors of this disease namely age, gender, tumor size, tumor grade, tumor stage, lymph node metastasis and survival of the patients.

\section{METHODS}

\section{Research design}

This cross-sectional study was performed at the departments of Pathology and Oncology, Ghaem Hospital, Mashhad, Iran. The diagnosis of GC was made between April 2004 and April 2010.

\section{Research subjects}

The study population consisted of patients with the diagnosis of intestinal type invasive GC 
(in which the tumor cells pass the stromal layer) who underwent gastrectomy at our medical center from 2004 to 2010. Inclusion criteria were patients having complete information of the required clinicopathological factors including age, gender, and immunohistochemistry (IHC) for CD10. Those with insufficient sample for H\&E and IHC staining or doubtful diagnosis or incomplete clinicopathological factors were excluded from study. To estimate the sample size, based on the study of Huang et al., ${ }^{(1)}$ we considered a confidence interval of 19 and confidence level of $99 \%$, the calculated sample size was 46 and for more confidence, 50 cases were entered into the study.

\section{Data collection}

A checklist of personal and paraclinical information, past medical history and prognosis was provided. The gathered variables include age, gender, tumor size, tumor grade, tumor stage, tumor location, lymphadenopathy, prognosis and CD10.

\section{Immunohistochemistry}

Subsequently, H\&E staining of the slides of all cases were controlled, paraffin blocks with sufficient tumoral and stromal tissue were taken and two pathologists checked and confirmed the IHC results of the samples to identify the expression of CD10. For the IHC study, $3 \times 4$ micron slides were provided and stained by rat monoclonal antibody [anti CD10, IgG1, 56C6 clone, NOVOCASTRA, UK]. We used renal cell carcinoma tissue as positive control and primary antibody as negative control. In terms of the percentage of staining, the cells were divided into 2 subsets: under $10 \%$ as negative and over $10 \%$ as positive.

\section{Statistical analysis}

The chi-square test was used to compare the studied variables between the two groups of patients with positive and negative CD10 expression. The Student t-test was applied to compare between these two groups. KaplanMeier analysis was used to compare overall survival time between negative CD10 and positive CD10 expression groups. Significance level was set at 0.05 . All analyses were performed using SPSS software (ver. 16.0, IBM).

\section{Ethical clearance}

The study protocol was fully supported by the Research Council Ethics Committee of our medical university (T-2852). Gastrectomy was indicated for the patients. The study was in conformity with the Declaration of Helsinki.

\section{Results}

Of 50 included patients, $43(86 \%)$ were male and seven (14\%) were female. Mean $( \pm \mathrm{SD})$ age of the patient was $65.2 \pm 9.4$ years (range, 43 to 80 years). Nine tumors were located in the cardia $(18 \%), 27$ in the body $(54 \%)$, two in the fundus (4\%), and 12 in the pyloric antrum (24\%). The maximum tumor diameter was between two and ten $\mathrm{cm}$ with an average of $5 \pm 2 \mathrm{~cm}$.

Three patients $(6 \%)$ had tumor stage I, eight cases $(16 \%)$ had stage II, 16 had stage IIIA, 12 cases had stage IIIB, and 11 patients had stage IIIC. In 20 samples (40\%), the tumor was well differentiated. Poorly differentiated tumors were observed in 12 samples (24\%) and in 18 cases (36\%), the tumor was moderately differentiated. Mean $( \pm \mathrm{SD})$ overall survival in the studied patients was $26.1 \pm 9.3$ months. There was no significance difference of age, gender, lymphadenopathy and tumor location by CD10 (Table 1).

\section{IHC staining}

Twenty-three samples (46\%) were positive for CD10 expression. Surrounded dysplastic mucus was positive in $5(10 \%)$ cases and stroma of normal tissue was negative. At the last followup, 41 patients $(82 \%)$ had died. 
Table 1. Distribution of age, gender, lymphadenopathy and tumor location by CD10

\begin{tabular}{lccc}
\hline & CD10 positive & CD10 negative & p value \\
\hline Age (years) & $66.4 \pm 9.1$ & $64.2 \pm 8.9$ & 0.435 \\
Gender & $19(44,2 \%)$ & $24(55,8 \%)$ & 0.681 \\
$\quad$ Male & $4(57.1 \%)$ & $3(42,9 \%)$ & \\
Female & & & \\
Lymph node involvement & $16(32 \%)$ & $22(44 \%)$ & 0.508 \\
$\quad+$ & $7(14 \%)$ & $5(10 \%)$ & 0.413 \\
- & & & \\
Tumor location & $5(10 \%)$ & $4(8 \%)$ & \\
Cardia & $13(26 \%)$ & $14(28 \%)$ & \\
Body & 0 & $2(4 \%)$ & \\
Fundus & $5(10 \%)$ & $7(14 \%)$ & \\
$\quad$ Pyloric antrum & & & \\
\hline
\end{tabular}

The mean age of patients with CD10expressing tumors was 64 years which was not different from the mean age of 27 patients with negative CD10 expression which was 66 years $(\mathrm{p}=0.435)$. Of 23 patients with positive CD10 expression, 19 were male and four were female $(\mathrm{p}=0.681)$.

No significant relationship was found between CD10 expression and tumor location $(\mathrm{p}=0.413)$. Likewise, no relationship was found between CD10 expression and lymph node metastasis $(p=0.508)$ (Table 2). However, a significant relationship was seen between CD10 expression and tumor grade $(\mathrm{p}=0.016)$, tumor stage $(\mathrm{p}=0.011)$, and survival $(\mathrm{p}=0.020)($ Table 2 ). Average overall survival in the $C D 10$ positive group was 16 months and in the CD10 negative group group 34 months (Log rank Mantel-Cox, $\mathrm{p}=0.020$ ) (Figure 1).

\section{DISCUSSION}

In our study, there was a significant correlation between CD10 expression and tumor differentiation as well as overall survival. However, age, gender, tumor location and lymph node metastasis seem not to be statically related to CD10 expression. Moreover, in our study in contrast to the results of a similar study, ${ }^{(1)}$ a statistically significant correlation was detected between tumor stage and CD10 expression.

Despite the reduction in the incidence of GC worldwide as seen in the recent years, this malignancy is still the fifth most common malignant tumor with high mortality rate. Generally, the prognosis of GC is poor despite observed improvements in surgical as well as adjuvant therapy of GC. These facts render it necessary to determine the prognosis of GC and to identify

Table 2. Relationship between tumor stage, tumor grade and survival with CD10 expression in patients with gastric adenocarcinoma $(\mathrm{n}=50)$

\begin{tabular}{|c|c|c|c|}
\hline & \multicolumn{2}{|c|}{ CD 10 expression } & \multirow{2}{*}{$p$ value } \\
\hline & Positive (n=23) & Negative $(n=27)$ & \\
\hline \multicolumn{4}{|l|}{ Tumor stage } \\
\hline I & 2 & 1 & 0.011 \\
\hline II & 2 & 6 & \\
\hline IIIA & 6 & 10 & \\
\hline IIIB & 9 & 3 & \\
\hline IIIC & 4 & 7 & \\
\hline \multicolumn{4}{|l|}{ Tumor grade } \\
\hline Poorly differentiated & 5 & 7 & 0.016 \\
\hline Moderately differentiated & 3 & 15 & \\
\hline Well differentiated & 15 & 5 & \\
\hline \multicolumn{4}{|l|}{ Survival } \\
\hline Alive & 1 & 8 & 0.020 \\
\hline Dead & 22 & 19 & \\
\hline
\end{tabular}




\section{Survival Functions}

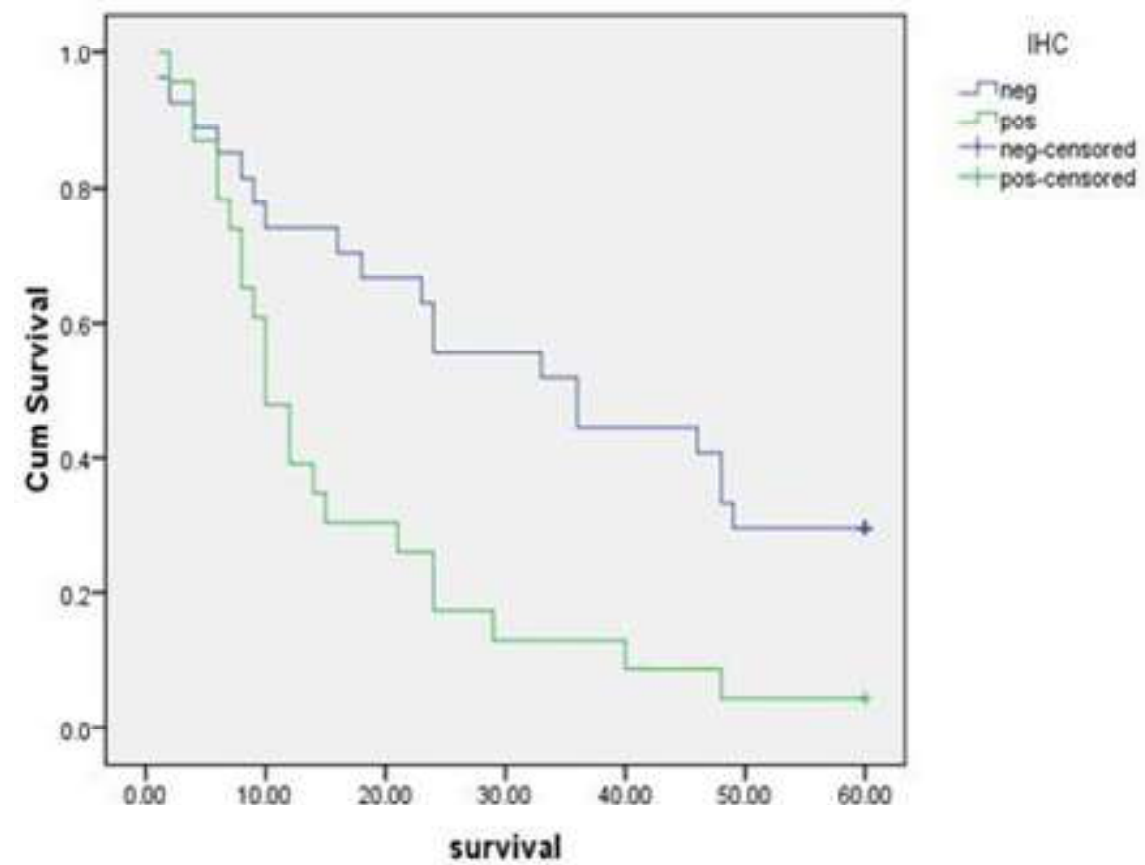

Figure 1. Overall survival curves for negative CD10 and positive CD10 expression groups among patients with gastric cancer

its prognostic factors, including histopathologic markers such as CD10, and any possible correlation between CD10 and other present prognostic factors namely, age, tumor location, tumor stage, and grade and lymph node metastasis. ${ }^{(12-14)}$ Recent studies of cancer research have been focusing on new targets being able to predict prognosis the prognosis or explain the invasive as well as the metastatic mechanism or highlight new strategies for treatment.

The CD10 antigen is a membrane glycoprotein that is normally expressed in early lymphoid progenitors and normal germinal center $\mathrm{B}$ cells. Therefore, CD10 expression has gained attention especially in B cell lymphoma. ${ }^{(15)}$ Furthermore, a study claimed that in stage I lung adenocarcinoma, tumoral CD10 correlates with high-grade histology and is an independent predictor of recurrence in intermediate-grade tumors. ${ }^{(16)}$

Another study on CD10 expression was on the cancer-associated fibroblasts (CAF) and their effects on colorectal cancer initiation and progression. CD10 expression was not detected in the normal colorectal mucosa. Significant correlation was found between CAF and high CD10 expression. ${ }^{(17)}$ It has been shown that extracellular proteinase may contribute to promotion of regulated growth factors and cytokines. These alterations can result in tumor progression. Since CD10 is structurally very similar to matrix metalloproteinase and stromelysin, it might facilitate cancer cell invasion and/or metastasis. The aim of one study was to determine the rate of CD10 expression in the stromal cells of invasive ductal breast carcinomas and the immunohistochemical aspects. Stromal CD10 expression in intraductal carcinoma (IDC) is closely correlated with invasion and metastasis and might play an important role in the pathogenesis of IDC. ${ }^{(18,19)}$ Finally another research pointed out that the CD10 antigen has been shown to be lost as an early event in prostate cancer. Loss of CD10 expression appears to be associated with increasing tumor grade in carcinoma of the prostate and this can potentially be useful in the stratification of such patients. ${ }^{(20)}$ 
A study by Kim et al. ${ }^{(21)}$ showed that there was no significant association between survival rate and the expression of other mucins (i.e. MUC2, MUC6, CD10) or mucin phenotypes.

Obviously, dozens of CD10 expression effects may be seen in different types of cancers, but GC is not one of them. Therefore, we intended to examine the correlation of CD10 expression with other clinicopathological and prognostic factors in GC.

The main limitation we had was the lack of enough cancerous tissue for staining which made us exclude some samples from our study. In this study, we showed that CD10 expression is associated with the aggressive biological behavior of the tumor, so it seems that using medications to reduce its expression would help overall survival to a large degree. In the future, some cohort studies in this regard will definitely help to reach a more effective conclusion.

\section{CONCLUSION}

This study demonstrated that CD 10 expression was correlated with tumor grade and survival rate. CD 10 expression can be used to predict outcomes in GC.

\section{ACKNOWLEDGMENTS}

The study was supported financially by the Vice Chancellor for Research of Mashhad University of Medical Sciences, Mashhad, Iran. The biospecimens for this study were provided by the surgeons of Ghaem Hospital. This study was the result of the thesis to obtain specialty degree in Pathology by Dr. Leila Takallou.

\section{CONFLICT OF INTEREST}

None.

\section{CONTRIBUTORS}

AHJ contributed to the conception of the work, conducting the study, revising the draft, approval of the final version of the manuscript, and of all aspects of the work. MKF contributed to drafting and revising the draft. LT contributed to conducting the study. NMR contributed in conception development and statistical analysis of the study. All authors read and approved the final manuscript.

\section{REFERENCES}

1. Karimi P, Islami F, Anandasabapathy S, et al. Gastric cancer: descriptive epidemiology, risk factors, screening, and prevention. Cancer Epidemiol Biomarkers Prev 2014;23:700-13. doi: 10.1158/1055-9965.EPI-13-1057.

2. Ferlay J, Shin HR, Bray F, et al. Estimates of worldwide burden of cancer in 2008: GLOBOCAN 2008. Int JCancer 2010;127:2893-917. doi: 10.1002/ ijc. 25516 .

3. Van Cutsem E, Sagaert X, Topal B, et al. Gastric cancer. Lancet 2016; 388: 2654-64. doi: 10.1016/ S0140-6736(16)30354-3.

4. Soerjomataram I, Lortet-Tieulent J, Parkin DM, et al. Global burden of cancer in 2008: a systematic analysis of disability-adjusted life-years in 12 world regions. Lancet 2012; 380: 1840-50. doi: 10.1016/S0140-6736(12)60919-2.

5. Maguer-Satta V, Besancon R, Bachelard-Cascales E. Concise review: neutral endopeptidase (CD10): a multifaceted environment actor in stem cells, physiological mechanisms, and cancer. Stem Cells 2011;29:389-96. doi: 10.1002/stem.592.

6. Oba J, Nakahara T, Hashimoto-Hachiya A, et al CD10-equipped melanoma cells acquire highly potent tumorigenic activity: a plausible explanation of their significance for a poor prognosis. PLoS One 2016;11:e0149285. doi: 10.1371/journal.pone. 0149285 .

7. Fukusumi T, Ishii H, Konno M, et al. CD10 as a novel marker of therapeutic resistance and cancer stem cells in head and neck squamous cell carcinoma. Br J Cancer 2014;111:506-14. doi: 10.1038/bjc.2014.289.

8. Salibay CJ, Rewerska J, Gupta S, et al Primary carcinosarcoma of the pancreas with CD10positive sarcoma component. J Investig Med High Impact Case Rep 2017;5:2324709617740906. doi: 10.1177/2324709617740906.

9. Murakami T, Yao T, Mitomi $\mathrm{H}$, et al. Clinicopathologic and immunohistochemical characteristics of gastric adenocarcinoma with enteroblastic differentiation. Gastric Cancer 2016;19:498-507. doi: 10.1007/s10120-015-0497-9. 
10. Ohnishi N, Takata K, Miyata-Takata T, et al. CD10 down expression in follicular lymphoma correlates with gastrointestinal lesion involving the stomach and large intestine. Cancer Sci. 2016;107:1687-95. doi: 10.1111/cas.13031.

11. Huang WB, Zhou XJ, Chen JY, et al. CD10positive stromal cells in gastric carcinoma: correlation with invasion and metastasis. Jpn J Clin Oncol 2005;35:245-50. doi:10.1093/jjco/ hyi076.

12. Feng F, Liu J, Wang F, et al. Prognostic value of differentiation status in gastric cancer. BMC Cancer 2018;18:865. doi: 10.1186/s12885-018-47800.

13. Giampieri R, Del Prete M, Cantini L, et al. Optimal management of resected gastric cancer. Cancer Manag Res 2018;10:1605-18. doi: 10.2147/ CMAR.S151552.

14. Zhang J, Gan L, Xu MD, et al. The prognostic value of age in non-metastatic gastric cancer after gastrectomy: a retrospective study in the U.S. and China. J Cancer 2018;9:1188-99. doi: 10.7150/ jca.22085.

15. Xu J, Medeiros LJ, Saksena A, et al. CD10-positive mantle cell lymphoma: clinicopathologic and prognostic study of 30 cases. Oncotarget 2018;9:11441-50. doi: 10.18632/oncotarget.23571.

16. Kadota K, Buitrago D, Lee MC, et al. Tumoral CD10 expression correlates with high-grade histology and increases risk of recurrence in patients with stage I lung adenocarcinoma. Lung Cancer 2015;89:329-36. doi: 10.1016/j.lungcan. 2015.06.003.
17. Zhu Y, Zheng JJ, Yang F, et al. Expression of CD10 in cancer-associated fibroblasts and its effect on initiation and progression of colorectal carcinoma. Chin J Pathol 2016;45:859-65. doi: 10.3760/cma.j. issn.0529-5807.2016.12.009.

18. Taghizadeh-Kermani A, Jafarian AH, Ashabyamin $\mathrm{R}$, et al. The stromal overexpression of CD10 in invasive breast cancer and its association with clincophathologic factors. Iran J Cancer Prev 2014;7:17-21.

19. Puri V, Jain M, Thomas S. Stromal expression of CD10 in invasive breast carcinoma and its correlation with ER, PR, HER2-neu, and Ki67. Int J Breast Cancer 2011, Article ID 437957, 4 pages. DOI: http://dx.doi.org/10.4061/2011/437957.

20. Kaur M, Verma S, Gupta R, et al. CD10 expression pattern in prostatic adenocarcinoma: Elucidation of differences between Gleason's grades. Malays J Pathol 2018 40:57-60.

21. Kim DH, Shin N, Kim GH, et al. Mucin expression in gastric cancer: reappraisal of its clinicopathologic and prognostic significance. Arch Pathol Lab Med 2013;137:1047-53. doi: 10.5858/arpa.2012-0193-OA. 